\title{
CONLEY INDEX FOR SET-VALUED MAPS: FROM THEORY TO COMPUTATION
}

\author{
TOMASZ KACZYNSKI \\ Département de mathématiques et d'informatique, Université de Sherbrooke \\ Sherbrooke J1K 2R1 (Qc), Canada \\ E-mail:kaczyn@dmi.usherb.ca
}

\begin{abstract}
Recent results on the Conley index theory for discrete multi-valued dynamical systems with their consequences for the computation of the index for representable maps are recapitulated. The terminology is simplified with respect to previous presentations, some superfluous hypotheses are abandoned and some conclusions are proved in a simpler way.
\end{abstract}

1. Introduction. Recent applications of the Conley index theory to study the chaotic dynamics given by Mischaikow, Mrozek and co-authors [9, 10, 11] involve numerical computations based on the concept of representable set-valued maps. Those computations motivated the generalization of the Conley index theory to set-valued maps in [4].

A continuous single-valued map whose dynamics is to be studied usually is not given by an explicit formula but it must be investigated numerically. Precise error estimates provide sets where the exact values of $f$ are located and so one obtains a multivalued map $F$ whose graph contains the graph of $f$. Such maps $F$ can be constructed so that they are finitely representable (e.g. their graphs are finite unions of cubes of a given grid in $\mathbb{R}^{n}$ ) and yet belong to the class of upper semicontinuous maps which share many nice properties with continuous maps. The clue for applying the set-valued maps lies in the fact that many of their properties are hereditary, i.e. they carry over from a set-valued map $F$ to any selection $f$ of $F$. By computing e.g. the index pair or the Conley index for $F$, we obtain an answer for $f$.

Applications of this technique became so popular that we are motivated to recapitulate in this paper the most important concepts and results related to the Conley index for set-valued maps. Most of the matter discussed in Sections 2 and 3 was presented in [12] for continuous maps, in $[4,5]$ and in subsequent expositions for set-valued maps

1991 Mathematics Subject Classification: Primary 58F35; Secondary 54C60, 54H20.

The author gratefully acknowledges the support from NSERC of Canada and FCAR of Quebec.

The paper is in final form and no version of it will be published elsewhere. 
but, as time passed, many improvements came about. Several definitions were simplified and some superfluous hypotheses abandoned. Only after having written [4], the authors realized that their result implies, as an easy consequence, the existence of index pairs which are stable under small perturbations of a map $f$. That, in turn, allowed to present simpler proofs of results of $[12,14]$ and [4] and to obtain other useful conclusions. In [6] an easier proof of the functoriality of the Conley index is presented. The existence of polyhedral index pairs for a map in $\mathbb{R}^{n}$ is deduced from [4] by Szymczak and algorithms for computing index pairs are given in $[13,16]$. At the end of Section 3 we present a much easier proof of the homotopy property of the Conley index than those given in $[12,14]$. In Section 4 we briefly discuss the application of the general theory to representable maps studied among others in $[1,11,13,16]$ and we comment on the issue of computing their homology which is investigated in $[1,7,8,11]$.

2. Isolating neighborhoods and index pairs. We first recall some terminology concerning set-valued maps $F: X \rightarrow \mathcal{P}(Y)$, where $X, Y$ are metric spaces and $\mathcal{P}(Y)$ is the set of all subsets of $Y$. The image $F(A)$ of $A \subset X$ by $F$, the graph $G(F)$ of $F$, the inverse $F^{-1}: Y \rightarrow \mathcal{P}(X)$ of $F$, and the effective domain $D(F)$ of $F$ are respectively defined by

$$
\begin{aligned}
& F(A):=\bigcup\{F(x): x \in A\}, \\
& G(F):=\{(x, y) \in X \times Y: y \in F(x)\}, \\
& F^{-1}(y):=\{x \in X: y \in F(x)\}, y \in Y, \\
& D(F):=\{x \in X: F(x) \neq \emptyset\} .
\end{aligned}
$$

Recall that $F$ is upper semicontinuous (usc) if and only if the set $\{x \in X: F(x) \subset U\}$ is open for any open $U \subset Y$ or, equivalently if $F^{-1}(A)=\{x \in X: F(x) \cap A \neq \emptyset\}$ is closed for any closed $A \subset Y$. It is known that a map $F: X \rightarrow \mathcal{P}(Y)$ with compact values is usc if and only if the following two conditions hold

1. $G(F)$ is closed,

2. $F(K)$ is compact for any compact $K \subset X$.

Hence the inverse $F^{-1}$ of an usc map $F$ is usc if and only if $F^{-1}(K)$ is compact for any $K \subset Y$. It is easily seen that the effective domain $D(F)$ of an usc map $F$ is closed.

Let now $(X, d)$ be a given locally compact metric space and $F: X \rightarrow \mathcal{P}(X)$ an usc map with compact values (empty values are allowed). Given $A \subset X$, we denote by $\bar{A}$ its closure, int $A$ its interior, $\partial A$ its boundary, and we put $B_{r}(A)=\{x \in X: d(x, A)<r\}$.

Given $x \in X$, an orbit (respectively, positive orbit, negative orbit) of $x$ for $F$ is a sequence $\left\{x_{n}\right\}_{n \in \mathbb{Z}}$ (respectively $\left\{x_{n}\right\}_{n \geq 0},\left\{x_{n}\right\}_{n \leq 0}$ ) such that

$$
x_{0}=x \quad \text { and } \quad x_{n+1} \in F\left(x_{n}\right)
$$

for all $n \in \mathbb{Z}$ (respectively, for all $n \geq 0, n<0$ ).

Note that if $f: X \rightarrow X$ is a single-valued continuous map and $F$ is defined by $F(x):=\overline{B_{\alpha}(f(x))}$ then $\left\{x_{n}\right\}_{n \in \mathbb{Z}}$ is an orbit for $F$ if and only if it is an $\alpha$-pseudo orbit for $f$ (cf. [2]).

Given a compact $N \subset X$, we define its invariant parts inv $N$ (respectively, $\operatorname{inv}^{+} N$, $\operatorname{inv}^{-} N$ ) to be the set of those $x \in N$ for which there exists an orbit (respectively, positive 
orbit, negative orbit) of $x$ for $F$ which is contained in $N$. It is easily seen that inv $N=$ $\operatorname{inv}^{+} N \cap \operatorname{inv}^{-} N$. Below, we give a slightly different description of these sets. Let $F_{N}$ : $N \rightarrow \mathcal{P}(N)$ be given by

$$
F_{N}(x):=F(x) \cap N, \quad x \in N .
$$

It is clear that $F_{N}$ is usc, moreover $F_{N}^{-1}$ is usc, which does not need to be true about $F^{-1}$. The iterates of $F_{N}$ which are recursively defined by $F_{N}{ }^{n+1}(x):=F_{N}\left(F_{N}{ }^{n}(x)\right)$ are usc and so are the iterates $F_{N}^{-n}$ of $F_{N}^{-1}$. We have the following

Proposition 2.1. Let $N \subset X$ be compact. Then

(a) $i n v^{+} N=\bigcap_{n \geq 0} D\left(F_{N}{ }^{n}\right)=\bigcap_{n \geq 0} F_{N}{ }^{-n}(N)$.

(b) $i n v^{-} N=\bigcap_{n \geq 0} D\left(F_{N}{ }^{-n}\right)=\bigcap_{n \geq 0} F_{N}{ }^{n}(N)$.

The first identity of (a) and (b) is proved in [4]. The second identity easily follows from the first one.

As an immediate consequence of Proposition 2.1, the sets inv ${ }^{+} N, \operatorname{inv}^{-} N$ and inv $N$ are compact.

Let $\operatorname{diam}_{N} F:=\sup \{\operatorname{diam} F(x): x \in N\}$ and $\operatorname{dist}(A, B):=\min \{d(x, y): x \in A$, $y \in B\}, A, B \subset X$. A compact subset $N \subset X$ is called an isolating neighborhood for $F$ if

$$
B_{\operatorname{diam}_{N} F}(\operatorname{inv} N) \subset \operatorname{int} N
$$

or, equivalently, if $\operatorname{dist}(\operatorname{inv} N, \partial N)>\operatorname{diam}_{N} F$. A set $S \subset X$ is called an isolated invariant set of $F$ if there exists an isolating neighborhood $N \subset X$ with $S=\operatorname{inv} N$. A pair $P=\left(P_{1}, P_{2}\right)$ of compact subsets $P_{2} \subset P_{1} \subset N$ is called an index pair for $F$ and $N$ if the following conditions are satisfied:

(a) $F\left(P_{i}\right) \cap N \subset P_{i}, i=1,2$,

(b) $F\left(P_{1} \backslash P_{2}\right) \subset N$,

(c) $\operatorname{inv} N \subset \operatorname{int}\left(P_{1} \backslash P_{2}\right)$.

The following theorem is proved in [4]:

THEOREM 2.1. Let $N$ be an isolating neighborhood for $F$ and $W$ a neighborhood of inv $N$ in $N$. Then there exists an index pair $P$ for $F$ and $N$ with $P_{1} \backslash P_{2} \subset W$.

We shall briefly outline the construction of $P$ discussed in [4]. Given $A \subset N$ we let

$$
F_{N}{ }^{+}(A):=\bigcup_{n \geq 0} F_{N}^{n}(A) .
$$

It is proved that one can find a compact neighborhood $A$ of inv $^{-} N$ in $N$ and an open neighborhood $U$ of $\operatorname{inv}^{+} N$ such that the pair $P=\left(P_{1}, P_{2}\right)$ given by

$$
P_{1}:=F_{N}{ }^{+}(A), \quad P_{2}:=F_{N}{ }^{+}\left(P_{1} \backslash U\right)
$$

is an index pair for $F$ and $N$ with $P_{1} \backslash P_{2} \subset W$. The difficulty of the proof lies in the fact that $F_{N}{ }^{+}$does not need to be usc. Nevertheless, $F_{N}{ }^{+}$behaves well on compact subsets of $N$ which either contain inv ${ }^{-} N$ or are disjoint with $\operatorname{inv}^{+} N$. When $F$ is a representable map, the construction is simplified and algorithms for computing $P$ are provided in [13, 15]. 
We shall now discuss isolating neighborhoods and index pairs for families of maps. In order to distinguish between different maps, we use the notation $\operatorname{inv}(N, F)$, inv ${ }^{-}(N, F)$, $\operatorname{inv}^{+}(N, F)$ for invariant parts of $N$ with respect to $F$. First, we have the following

Proposition 2.2. Let $F: X \rightarrow \mathcal{P}(X)$ and $G: X \rightarrow \mathcal{P}(X)$ be two compact valued usc maps with $F \subset G$, i.e. $F(x) \subset G(x)$ for all $x \in X$. Let $N$ be an isolating neighborhood for $G$ and $P$ an index pair for $G$ and $N$. Then

1) $i n v^{+}(N, F) \subset i n v^{+}(N, G)$ and $i n v^{-}(N, F) \subset i n v^{-}(N, G)$,

2) $N$ is an isolating neighborhood for $F$,

3) $P$ is an index pair for $F$.

Although the proof is simple routine verification, Proposition 1.1 has very deep consequences. It states that the property of being an isolating neighborhood or an index pair is hereditary, so it carries over from a multivalued map to its selections.

Let now $\Lambda$ be an interval in $\mathbb{R}$ and $F: X \times \Lambda \rightarrow \mathcal{P}(X)$ an usc map with compact values. We set $F_{\lambda}: X \rightarrow \mathcal{P}(X), F_{\lambda}(x):=F(x, \lambda)$. The following lemmas are proved in $[4,5]$ :

Lemma 2.1. Let $N \subset X$ be compact. Then the maps $\lambda \rightarrow i n v^{+}\left(N, F_{\lambda}\right), \lambda \rightarrow i n v^{-}\left(N, F_{\lambda}\right)$ and $\lambda \rightarrow \operatorname{inv}\left(N, F_{\lambda}\right)$ are usc.

Lemma 2.2. Let $\lambda_{0} \in \Lambda$ and let $N$ be an isolating neighborhood for $F_{\lambda_{0}}$. Then $N$ is an isolating neighborhood for $F_{\lambda}$, for all $\lambda$ sufficiently close to $\lambda_{0}$.

The conclusion cf Lemma 2.1 would not be true for index pairs. In [14] a counterexample is given. However, we derive below the existence of locally constant index pairs as a simple consequence of Theorem 2.1 and Proposition 2.2.

Corollary 2.1. Let $N$ be an isolating neighborhood for $F_{\lambda}$, for all $\lambda \in \Lambda$ (that may be assumed in view of Lemma 2.2). Then, for each $\lambda_{0} \in \Lambda$ there exists an index pair $P$ for $F_{\lambda_{0}}$ which also is an index pair for $F_{\lambda}$ provided $\lambda$ is sufficiently close to $\lambda_{0}$.

Proof. Let $G_{r}(x):=\overline{B_{r}\left(F_{\lambda_{0}}(x)\right)}, r \in[0,1]$. Then $G: X \times[0,1] \rightarrow \mathcal{P}(X)$ is usc with compact values and by Lemma 2.2 applied for $G$ there exists $0<\varepsilon \leq 1$ such that $N$ is an isolating neighborhood for $G_{\varepsilon}$. Since $F$ is usc, there exists $\delta>0$ such that $F_{\lambda} \subset G_{\varepsilon}$ provided $\left|\lambda-\lambda_{0}\right| \leq \delta$. By Proposition 2.2, $P$ is an index pair for all those $F_{\lambda}$.

Corollary 2.2. Let $f: X \rightarrow X$ be a continuous map and $N$ an isolating neighborhood for $f$. Then there exists an index pair $P$ for $f$ and $N$ which is invariant under all sufficiently small $C^{0}$ perturbations of $f$, i.e. continuous maps $f_{\varepsilon}: X \rightarrow X$ such that $d\left(f(x), f_{\varepsilon}(x)\right) \leq \varepsilon$ for all $x \in N$.

Proof. Put $F_{\lambda}(x):=\overline{B_{\lambda}(f(x))}$ and apply Corollary 2.1. If $\varepsilon>0$ is sufficiently small then both $f$ and $f_{\varepsilon}$ are selections of $F_{\lambda}$ so Proposition 2.2 may be used as before.

3. Cohomology of acyclic-valued maps and the Conley index. Let $X, Y$ be locally compact metric spaces. In what follows, $H^{*}($.$) stands either for the \check{C}$ ech cohomology or the Alexander-Spanier cohomology with field coefficients. In general, we need here any cohomology functor satisfying the homotopy, exactness, excision, and dimension 
axioms in $[15$, Ch. 5 , Sect. 4] and the continuity property [15, Ch. 6, Sect. 6]. In applications to representable maps discussed in $[1,13]$, the only spaces appearing in the study are finite polyhedra and, in that case, all what is said below carries over to any homology or cohomology theory with either field or integer coefficients.

Let $\mathcal{A c}(X)$ stand for the family of all non-empty compact sets $K$ of $X$ which are acyclic, i.e. $\tilde{H}^{*}(K)=0$, where $\tilde{H}^{*}(K)$ is the reduced cohomology. We recall from $[15$, Ch. 6, Sect. 9] the Vietoris-Begle Theorem:

THEOREM 3.1. Let $f: X \rightarrow Y$ be a continuous map satisfying the following conditions

1) $f$ is proper, i.e. $f^{-1}(K)$ is compact for any compact $K \subset Y$,

2) $f$ is surjective,

3) $f^{-1}(y)$ is acyclic for all $y \in Y$.

Then $f$ induces an isomorphism $H^{*}(f): H^{*}(Y) \rightarrow H^{*}(X)$.

For simplicity of notation, we put $f^{*}=H^{*}(f)$. Let now $F: X \rightarrow \mathcal{A c}(Y)$ be an usc map. Then $F$ induces a map $F^{*}: H^{*}(Y) \rightarrow H^{*}(X)$ in cohomology as follows. Let $p: G(F) \rightarrow X$ and $q: G(F) \rightarrow Y$ be canonical projections from the graph of $F$. Then $p$ satisfies the hypotheses of the Vietoris-Begle Theorem, so $p^{*}: H^{*}(X) \rightarrow H^{*}(G(F))$ is an isomorphism. One defines

$$
F^{*}:=\left(p^{*}\right)^{-1} q^{*} .
$$

In case when $f: X \rightarrow Y$ is continuous and $F=\{f\}, p$ is a homeomorphism, so the above definition coincides with the usual one. When $F$ is set-valued and $f$ is a continuous selection of $F$, then $f^{*}=F^{*}$. A very simple proof of that fact (for homology) is presented in [1] and, by the same arguments, we get

Proposition 3.1. Let $F, G: X \rightarrow \mathcal{A c}(Y)$ be usc maps such that $F \subset G$. Then $F^{*}=G^{*}$.

All what is said above clearly extends to maps $F:(X, A) \rightarrow(\mathcal{A} c(Y), \mathcal{A} c(B))$ on pairs of spaces, i.e. maps $F: X \rightarrow \mathcal{A} c(Y)$ with $F(A) \subset B$, where $A$ is a closed subset of $Y$. We obtain maps $F^{*}: H^{*}(Y, B) \rightarrow H^{*}(X, A)$ induced in the relative cohomology.

Proposition 3.2 (cf. [4]). If $P$ is an index pair for $F$ and $N$ then

a) $\left(P_{1}, P_{2}\right) \subset\left(P_{1} \cup F\left(P_{2}\right), P_{2} \cup F\left(P_{2}\right)\right) \subset\left(P_{1} \cup(X \backslash\right.$ int $N), P_{2} \cup(X \backslash$ int $\left.N)\right)$,

b) the above inclusions induce isomorphisms in relative cohomology.

We put $T(P):=\left(P_{1} \cup(X \backslash \operatorname{int} N), P_{2} \cup(X \backslash \operatorname{int} N)\right)$, let $i_{P}: P \rightarrow T(P)$ be the inclusion map and $F_{P}: P \rightarrow \mathcal{A c}(T(P))$ be the restriction of $F$. The endomorphism $I_{P}$ of $H^{*}\left(P_{1}, P_{2}\right)$ given by

$$
I_{P}:=F_{P}^{*} \circ\left(i_{P}^{*}\right)^{-1}
$$

is called an index map of $F$ associated with $P$. In some expositions $i_{P}$ is defined as the first inclusion in $(a)$ only. The advantage of going further to $T(P)$ is that given different maps sharing the same index pair $P, T(P)$ does not depend on the map.

There are now several ways $[12,14,17]$ of defining the Conley index of an isolated invariant set $S=\operatorname{inv} N$ of which the definition given in [12] via the Leray reduction described below is the most convenient for computation. 
Set $E:=H^{*}(P)$ and $T:=I_{P}$. The generalized kernel of $T$ is

$$
\operatorname{gKer} T:=\bigcup_{n \geq 1} \operatorname{Ker} T^{n}
$$

Since $\operatorname{Ker} T^{n} \subset \operatorname{Ker} T^{n+1}$ for all $n \geq 1, \operatorname{gKer} T$ is a vector subspace of $E$ and since $T(\operatorname{gKer} T) \subset \operatorname{gKer} T$, the quotient map $T^{\prime}:=[T]: E^{\prime} \rightarrow E^{\prime}$, where $E^{\prime}=E / \operatorname{gKer} T$, is well defined. Note that $T^{\prime}$ is a monomorphism. In applications, $E^{\prime}$ is finite dimensional and then $T^{\prime}$ is an isomorphism. In general, we restrict $T^{\prime}$ to the subspace $E^{\prime \prime}=\operatorname{gIm} T^{\prime}$, where $\operatorname{gIm} T^{\prime}$, the generalized image of $T^{\prime}$ is

$$
E^{\prime \prime}=\operatorname{gIm} T^{\prime}=\bigcap_{n \geq 0} \operatorname{Im}\left(T^{\prime}\right)^{n}
$$

Since $T^{\prime}\left(E^{\prime \prime}\right)=E^{\prime \prime}$, the restriction $T^{\prime \prime}=T^{\prime} E^{\prime \prime}: E^{\prime \prime} \rightarrow E^{\prime \prime}$ is a well defined automorphism of $E^{\prime \prime}$. The Leray reduction of the pair $(E, T)$ is the pair $L(E, T):=\left(E^{\prime \prime}, T^{\prime \prime}\right)$ consisting of a graded vector space and its automorphism. We now define the Conley index of $S$ by

$$
\operatorname{Con}^{*}(S)=\left(C H^{*}(S), \mathcal{X}^{*}(S)\right):=L\left(H^{*}(P), I_{P}\right) .
$$

It is proved in [4] that this definition does not depend on the choice of an isolating neighborhood $N$ of $S$ of an index pair $P$ for $F$ and $N$. If $F$ is a single-valued continuous map generated by a flow then $I_{P}$ is the identity, so $L\left(H^{*}(P), I_{P}\right)=\left(H^{*}(P), I\right)$.

We shall now prove the homotopy property for the Conley index. We recall from $[3]$ the following

Theorem 3.2. Let $F: X \times[0,1] \rightarrow \mathcal{A} c(Y)$ be an usc map. Then $F_{\lambda}^{*}: H^{*}(Y) \rightarrow$ $H^{*}(X)$ does not depend on the choice of $\lambda \in[0,1]$.

Actually, the above homotopy theorem is discussed in [3] for homology but it naturally extends to cohomology.

Corollary 3.1. Let $F: X \times[0,1] \rightarrow \mathcal{A} c(Y)$ be an usc map and $N$ an isolating neighborhood for all $F_{\lambda}, \lambda \in \Lambda$ (due to Lemma 2.2 this assumption is not too restrictive). Put $S_{\lambda}:=\operatorname{inv}\left(N, F_{\lambda}\right)$. Then $\operatorname{Con}^{*}\left(S_{\lambda}\right)$ does not depend on $\lambda$.

Proof. Take arbitrary $\lambda_{0} \in[0,1]$. By Corollary 2.1 there exists $\delta>0$ and a pair $P=\left(P_{1}, P_{2}\right)$ which is an index pair for $F_{\lambda}$ provided $\left|\lambda-\lambda_{0}\right| \leq \delta$. By Theorem $3.2,\left(F_{\lambda}\right)_{p}^{*}$ is independent of those $\lambda$, so is $\mathrm{i}_{P}$ and, consequently, the index map $I_{P}$ is the same for all $\lambda$ with $\left|\lambda-\lambda_{0}\right| \leq \delta$. Hence $\lambda \rightarrow \operatorname{Con}^{*}\left(S_{\lambda}\right)$ is locally constant and, since $[0,1]$ is connected, it is constant.

4. Application to representable maps. The general notions of grids, representable sets, and representable maps are presented with all details in [13]. In this paper we concentrate our attention on cubic grids in $X=\mathbb{R}^{n}$ discussed in [1].

The cubic grid $\mathcal{E}_{k}$ of mesh $\frac{1}{k}, k=0,1,2, \ldots$ is the collection of all cubes $e \in \mathbb{R}^{n}$ of the form $e=\frac{1}{k}\left(x+I_{1} \times I_{2} \times \ldots \times I_{n}\right)$ where $x \in \mathbb{Z}^{n}$ and $I_{j}$ is either the open interval $] 0,1[$ or singleton $\{0\}$ or $\{1\}$.

The elements of $\mathcal{E}_{k}$ are called elementary cubes. Note that $\mathbb{R}^{n}$ decomposes as the disjoint union of $e \in \mathcal{E}_{k}$. There are many advantages of such decompositions over simpli- 
cial triangulations, for example, the product of two elementary cubes is an elementary cube which is not true about simplexes. A grid $\mathcal{E}_{l}$ is a subgrid of $\mathcal{E}_{k}$ if $k \mid l$ ( $k$ divides l). A subset $X$ of $\mathbb{R}^{n}$ is said to be representable over $\mathcal{E}_{k}$ or briefly, $k$-representable if there exists a finite $\mathcal{E}^{\prime} \subset \mathcal{E}_{k}$ such that $X=\bigcup \mathcal{E}^{\prime}$ and $X=\bar{X}$. In particular, $X$ is a finite polyhedron. The set $\mathcal{E}^{\prime}$ is uniquely defined and denoted by $\mathcal{E}_{k}(X)$. The family of all $k$-representable polyhedra in $\mathbb{R}^{n}$ is denoted by $\mathcal{R}_{k}$. The following notation is adopted for any $X \in \mathcal{R}_{k}$ :

$$
\begin{aligned}
& \mathcal{E}_{k}^{q}(X):=\left\{e \in \mathcal{E}_{k}: \operatorname{dim} e=q\right\} \text { and } \mathcal{E}_{k}^{q}(X)=\mathcal{E}_{k}(X) \cap \mathcal{E}^{q}, \\
& \mathcal{E}_{k}{ }^{\max }(X):=\left\{\sigma \in \mathcal{E}_{k}: \sigma \text { is not a proper face of any } e \in \mathcal{E}_{k}(X)\right\}, \\
& \mathrm{o}_{k}(X):=\bigcup\left\{e \in \mathcal{E}_{k}: \bar{e} \cap X \neq \emptyset\right\}, \\
& \text { st }_{k}(X):=\overline{\mathrm{o}_{k}(X)}=\bigcup\left\{\bar{e}: e \in \mathcal{E}_{k}, \bar{e} \cap X \neq \emptyset\right\} .
\end{aligned}
$$

Let $X \in \mathcal{R}_{k}$. A set-valued map $F: X \rightarrow \mathcal{R}_{k}$ is representable over $\mathcal{E}_{k}$ or, briefly, $k$-representable if its graph $G(F)$ is closed and $F$ is constant on elementary cubes, i.e. $F(x)=F(y)$ whenever $x, y \in e, e \in \mathcal{E}_{k}(X)$. It follows that $F$ is usc and that $G(F)$ is a $k$-representable set in the product space $\mathbb{R}^{2 n}$.

A $k$-representable map $F$ is called admissible if

i) $F(\sigma)$ is convex for all $\sigma \in \mathcal{E}_{k}^{\max }(X)$,

ii) $F(e)=\bigcup\left\{F(\sigma): e\right.$ is a face of $\left.\sigma, \sigma \in \mathcal{E}_{k}^{\max }(X)\right\}$, for all $e \in \mathcal{E}_{k}(X)$,

iii) $\mathcal{A}(e):=\bigcap\left\{F(\sigma): e\right.$ is a face of $\left.\sigma, \sigma \in \mathcal{E}_{k}(X)\right\} \neq \emptyset$, for all $e \in \mathcal{E}_{k}(X)$.

The above conditions imply that $F(e)$ is star-shaped and hence acyclic for all $e \in$ $\mathcal{E}_{k}(X)$. If a representable map $F$ admits a continuous selection $f$ then (iii) automatically is satisfied since $f(e) \subset \mathcal{A}(e)$. Conversely, any admissible map has a continuous piece-wise linear selection.

Let $X \in \mathcal{R}_{k}$ and $f: X \rightarrow \mathbb{R}^{n}$ be a continuous map. Any $k$-representable admissible map $F: X \rightarrow \mathcal{R}_{k}$ with $f \in F$ is called a $k$-representable collar of $f$. There exists one with the smallest values which is called the $k$-representation of $f$ and denoted by $F_{f, k}: X \rightarrow \mathcal{R}_{k}$.

It is verified that, for any continuous map $f: \mathbb{R}^{n} \rightarrow \mathbb{R}^{n}$ and an isolating neighborhood $M$ for $f$ one can find $k$ and an isolating neighborhood $N \in \mathcal{R}_{k}$ for $f$. Next, one can find $l$ such that $k \mid l$ and if $F=F_{k, l}: N \rightarrow \mathcal{R}_{l}$ then $\operatorname{diam}_{N} F<\operatorname{dist}(\operatorname{inv} N, \partial N)$, so that $N$ is an isolating neighborhood for $F$. Thus the study of the Conley index for $f$ reduces to the study of Conley index for $F$. The definition of the index pair for $f$ and $N$ simplifies a lot in that case. First, $F_{N}^{n}(N) \in \mathcal{R}_{l}$ for all $n \in \mathbb{Z}$ thus the intersections in Proposition 2.1 are over a finite family of representable sets and $\operatorname{inv}^{-} N, \operatorname{inv}^{+} N \in \mathcal{R}_{l}$. It is proved in [16] that one may define

$$
P_{1}:=\operatorname{st}_{m}\left(\operatorname{inv}^{-} N\right), \quad P_{2}:=P_{1} \backslash \mathrm{o}_{m}\left(\operatorname{inv}^{+} N\right),
$$

where $m>l, l \mid m$. As a consequence, $P_{1}, P_{2} \in \mathcal{R}_{m}$. Algorithms for verifying that a given $N \in \mathcal{R}_{l}$ is an isolating neighborhood for $F$ and for computing a representable index pair are given in $[13,16]$. The next goal is to compute $\operatorname{Con}^{*}(S)$ for an admissible map $F$ and a representable index pair $P$. But this reduces to computation of the map induced in cohomology. 
Given $X \in \mathcal{R}_{m}$, let $C(X, m)=\left\{C_{q}(X, m)\right\}_{q \in \mathbb{Z}}$ be the finitely generated free chain complex, where $C_{q}(X, m)$ is generated by $\mathcal{E}_{m}^{q}(X)$ for all $q\left(C_{q}(X, m)=0\right.$ if $q<0$ or $\left.q>n\right)$. We refer to [1] for the definition of the boundary map $\partial_{q}: C_{q}(X, m) \rightarrow C_{q-1}(X, m)$. $C(X, m)$ is called a cubical chain complex and its homology is isomorphic to the simplicial homology of $X$. We recall from [1] the following

Theorem 4.1. Let $X, Y \in \mathcal{R}_{m}$ and let $F: X \rightarrow \mathcal{P}(Y) \cap \mathcal{R}_{m}$ be an admissible $m$ representable map. Then there exists a chain map $\varphi: C(X, m) \rightarrow C(Y, m)$ with the property

$$
\left|\varphi_{q} \sigma\right| \subset \mathcal{A}(\sigma) \quad \text { for all } \sigma \in \mathcal{E}_{m}^{q}(X),
$$

where $\left|\varphi_{q} \sigma\right|$ is the support of the chain $\varphi_{q} \sigma$. Moreover,

i) Any two chain maps with the above property are chain homotopic,

ii) $\varphi_{*}=F_{*}$ (and so $\left.\varphi^{*}=F^{*}\right)$

In [1], an explicit construction of the chain map $\varphi$ is provided. An algorithm for computing $\varphi$ is in progress.

\section{References}

[1] M. AlLiLI and T. KACZYNSKI, An algorithmic approach to the construction of homomorphisms induced by maps in homology, Trans. Amer. Math. Soc., to appear.

[2] R. Bowen, Equilibrium States and The Ergodic Theory of Anosov Diffeomorphisms, Lecture Notes in Math. (470), Springer, 1975.

[3] L. GóRniewicz, Homological Methods in Fixed Point Theory of Multi-Valued Maps, Dissertationes Math. (129), PWN, Warsaw, 1976.

[4] T. KaCZYNSKi and M. MrozeK, Conley index for discrete multivalued dynamical systems, Topology Appl. 65 (1995), 83-96.

[5] T. Kaczynski and M. Mrozek, Stable index pairs for discrete dynamical systems, Canad. Math. Bull. 40 (4) (1997), 448-455.

[6] T. KaCZynski and M. Mrozek, Connected simple systems and the Conley functor, Topol. Meth. in Nonlin. Anal. 10 (1) (1997), 183-193.

[7] T. Kaczynski, M. MrozeK and M. ŚlusareK, Homology computation by reduction of chain complexes, Computers \& Math. Appl. 35 (4) (1998), 59-70.

[8] W. Kalies, K. Mischaikow and G. Watson, Cubical approximation and computation of homology, this volume.

[9] K. Mischaikow and M. MrozeK, Isolating neighborhoods and chaos, Japan J. Ind. and Appl. Math. 12 (1995), 205-236.

[10] K. Mischaikow and M. Mrozek, Chaos in the Lorenz equations: a computer assisted proof, Bull. Amer. Math. Soc. 32 (1995), 66-72.

[11] K. Mischaikow, M. Mrozek, J. Reiss and A. Szymczak, From time series to symbolic dynamics: an algebraic topological approach, preprint.

[12] M. Mrozek, Leray functor and cohomological index for discrete dynamical systems, Trans. Amer. Math. Soc. 318 (1990), 149-178.

[13] M. MrozeK, An algorithmic approach to the Conley index theory, preprint. 
[14] J. W. RobBin and D. SAlamon, Dynamical systems, shape theory and the Conley index, Ergodic Theory Dynamical Systems 8 (1988), 375-393.

[15] E. H. Spanier, Algebraic Topology, McGraw-Hill, 1966.

[16] A. Szymczak, A combinatorial procedure for finding isolating neighborhoods and index pairs, Proc. Royal Soc. Edinburgh Sect. A 127 (5) (1997), 1075-1088.

[17] A. Szymczak, The Conley index for discrete semidynamical systems, Topology Appl. 66 (3) (1995), 214-240. 